INGENIERÍA MECÁNICA

\title{
Uso de la Teoría de Solución de Problemas Inventivos (TRIZ) en el análisis de productos de apoyo a la movilidad para detectar oportunidades de innovación
}

MECHANICAL ENGINEERING

\section{Use of the Theory of Inventive Problem Solving (TRIZ) in the analysis of assistive products for personal mobility in order to detect innovation opportunities}

\author{
Jaime A. Aguilar-Zambrano*^, Manuel V. Valencia*, Manuel F. Martínez*, \\ Carlos A. Quiceno*, Claudia M. Sandoval* \\ *Grupo de Automática y Robótica, *Departamento de Electrónica y Ciencias de la Computación \\ * Pontificia Universidad Javeriana, Cali Colombia \\ §jaguilar@javerianacali.edu.co,mvalencia@javerianacali.edu.co,manolojethro@hotmail.com, \\ kiceno@gmail.com, sandoval.claudia@javeriana.edu.co
}

(Recibido: Marzo 24 de 2011 - Aceptado: Mayo 11 de 2012 -Versión Final: Junio 20 de 2012)

\begin{abstract}
Resumen
Se presenta una estrategia para detectar oportunidades de innovación en el diseño de productos de apoyo a la movilidad con la aplicación de las Leyes de Evolución de los Sistemas Técnicos. Estas leyes están enunciadas en la Teoría de la Solución de Problemas Inventivos: TRIZ. Los productos de apoyo pertenecen a la Clase 12 dentro de la norma UNE-EN ISO 9999 y el análisis se hace en forma conjunta con ingenieros y diseñadores. El estudio propone una forma novedosa de modelar los productos de apoyo como un sólo sistema hombre-máquina. Los resultados encontrados muestran oportunidades de innovación en: los sistemas de transmisión, el control del sistema, el mejoramiento del sistema de frenado, el aprovechamiento energético, la mejora en los niveles de accesibilidad del sistema al medio, y el acople usuario-dispositivo.
\end{abstract}

Palabras Claves:Innovación tecnológica, Leyes de Evolución Técnica, Productos de apoyo, Trabajo multidisciplinar, TRIZ.

\begin{abstract}
This paper presents a way of detecting innovation opportunities for assistive products for personal mobility applying the laws of technical evolution. These laws are formulated in the theory of inventive problem solving, TRIZ. The products belong to class 12 of UNE-EN ISO 9999 standard and the analysis is made in conjunction with engineers and industrial designers. This study proposes an innovative way to represent the technical system of this type of mobility aid products as a whole, this is, as a machine-human integral unit. The results obtained show innovation opportunities in the following fields: optimization of transmission systems, movement control and brake system, high efficiency energy use and improvement in the accessibility levels, both, from user to product and from product to environment.
\end{abstract}

Keywords: Innovation, laws of technical evolution, mobility aids, multidisciplinary approach, TRIZ. 


\section{Introducción}

Existen múltiples definiciones en torno al concepto de innovación, por ejemplo J. Pavón y R. Goodman (1981) afirman que: " Innovación es el conjunto de actividades, inscritas en un determinado período de tiempo y lugar, que conducen a la introducción con éxito en el mercado, por primera vez, de una idea en forma de nuevos o mejores productos, servicios o técnicas de gestión y organización". El Manual de Oslo se acoge a una definición que afirma que "una innovación es la implementación de una nueva o significante mejora de un producto (bien o servicio), un nuevo proceso, un nuevo método de mercado o un nuevo método de organización en las prácticas de negocio, en la organización del sitio de trabajo o en las relaciones externas" (Dutta 2005). Por su parte, otros autores sugieren que la innovación consiste en detectar necesidades y características de mejoramiento en productos del mercado para que, a través del uso del conocimiento científico, se ofrezcan unos nuevos $u$ optimizados que satisfagan las necesidades de los usuarios (Ciruello et al. 2008). Lo anterior implica hacer un análisis de las necesidades de los usuarios y utilizar el conocimiento científico para proveer nuevas soluciones.

Para el proceso de detectar oportunidades de innovación existen aproximaciones tanto desde el mercado como desde la ingeniería que en ocasiones no se complementan. Desde el mercado se utilizan estrategias como QFD y Benchmarking, entre otras, que priorizan la voz del usuario (Terninko 1997); por su parte, la ingeniería se concentra en el análisis y desarrollo de los aspectos técnicos del producto, pero en ocasiones se desconoce al usuario y su contexto de aplicación (Pugh 1991). Dada esta situación, en este artículo se presenta una aproximación integral del producto que considera la función que desempeña el producto en su interacción con el usuario; esto se hace mediante la aplicación del análisis de la evolución de los Sistemas Técnicos, el cual forma parte de la Teoría de Solución de Problemas Inventivos, TRIZ.

TRIZ es un conjunto de herramientas para fomentar la invención, desarrolladas por el equipo de trabajo de Genrich Altshuller en la antigua Unión Soviética desde 1946. A partir de los años 80 , la teoría se expandió a nivel mundial y actualmente se utiliza por múltiples empresas, integrándose efectivamente con otras estrategias de diseño (Moehrle 2005). La fuente de trabajo de TRIZ más conocida es la revisión de más de dos millones de patentes, y sus conceptos principales son: Contradicciones, Idealidad y Evolución de los Sistemas Técnicos. Actualmente, el trabajo investigativo alrededor de la teoría se orienta, entre otros, hacia el uso de principios inventivos derivados de la biología (Vincent et al. 2005), el trabajo multidisciplinar (Gonzalez-Cruz et al. 2008), el trabajo sinérgico con otras estrategias de diseño y creatividad (Kang 2004) (Aguilar et al. 2008).

Dentro de TRIZ, la Evolución en los Sistemas Técnicos es una técnica de análisis del comportamiento que exhiben los productos en el tiempo, de forma similar a los seres vivos. La evolución es la tendencia en el desarrollo de los productos para alcanzar la idealidad, que es la tendencia de un sistema (producto) a desempeñar su función sin consumir materia y energía, mediante la mejora continua de su desempeño. Para el análisis de la evolución de un producto es necesario representarlo como un Sistema Técnico (ST), compuesto por cuatro elementos: motor, transmisión, herramienta y control, con el fin de verificar el cumplimiento de las ocho leyes propuestas por Altshuller, (Cavallucci \& Weill 2001). TRIZ tiene otras técnicas para el diseño del producto, como la matriz de contradicciones, el análisis de nueve ventanas y el análisis camposustancia. En este artículo se utiliza el proceso de análisis de evolución de los sistemas técnicos para el análisis del problema y los resultados se convierten en insumo para el proceso de diseño.

El análisis de Evolución de los ST se ha aplicado a productos industriales (Mann 2002 y 2004), pero no se reportan casos para productos de apoyo. Estos tienen como una de sus finalidades compensar las deficiencias en las funciones fisiológicas de los sistemas corporales que presentan las personas en situación de discapacidad. En este artículo se presenta una forma novedosa de modelar los productos de apoyo como Sistemas Técnicos y su posterior 
análisis de evolución aplicado a los productos de la Clase 12 de la norma UNE-EN ISO 9999, (AENOR 2007).

El artículo se encuentra organizado en cinco secciones: la primera, introduce la teoría TRIZ junto con las Leyes de Evolución de los ST para identificar oportunidades de innovación; la segunda, describe el método de aplicación de las leyes en productos de apoyo para movilidad personal; la tercera, presenta los resultados obtenidos en el análisis de evolución para favorecer la innovación de este tipo de productos; finalmente, se presentan las conclusiones.

\section{La Teoría de Solución de Problemas}

\section{Inventivos, TRIZ, para la identificación de oportunidades de innovación en Sistemas Técnicos}

Las oportunidades de innovación surgen a partir de la identificación de elementos de desarrollo en los productos para su mejora continua con base en la aplicación del conocimiento. La teoría TRIZ provee una herramienta para la evaluación del estado de evolución de un producto, a partir de la cual se pueden identificar estas oportunidades.
Cavalluci (2002) realiza una clasificación del trabajo de Altshuller en torno a TRIZ de la siguiente forma: nociones esenciales, herramientas para prevenir la inercia psicológica y herramientas de solución como se muestra en la Figura 1; los conceptos claves evidentes en esta clasificación son: la idealidad, ARIZ, las contradicciones, el análisis campo-sustancia, las Leyes de Evolución de los Sistemas Técnicos y la base de conocimiento de los efectos y principios inventivos. Cuando se aborda un problema inventivo desde la teoría TRIZ, se conduce el proceso hacia la búsqueda estructurada de soluciones más específicas, pues el problema inicial se convierte en uno general de diseño TRIZ (véase Figura 2), el cual se basa en el análisis y clasificación de un gran número de problemáticas en diversos campos de la ingeniería. El potencial de la teoría radica en la habilidad para sugerir soluciones desde diferentes, o aparentemente, campos inconexos y trasladarlas a un problema de diseño que se caracteriza por la innovación (Salamatov 1999).

\subsection{Las Leyes de Evolución de los Sistemas Técnicos en TRIZ}

Un Sistema Técnico (ST) es un producto artificial que desempeña una función, el cual toma que toma

\section{Fuentes}

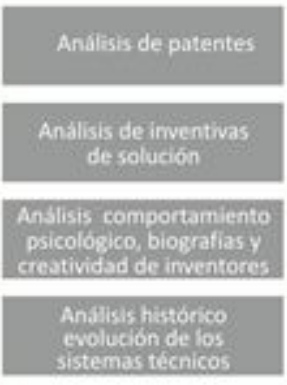

Ansisix de motodo: existentes y recnicas

Anślisis de literatura cientifica y tecrica

\section{Análisis}

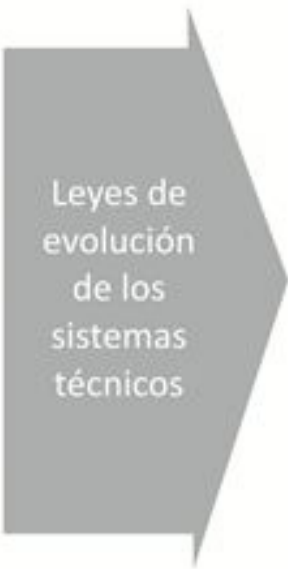

\section{Clasificación}
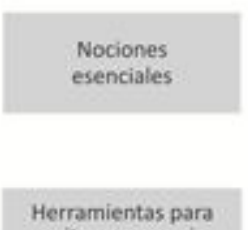

Herramientas para
evitar o prevenir
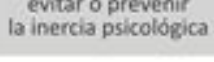

Herramientas de solución
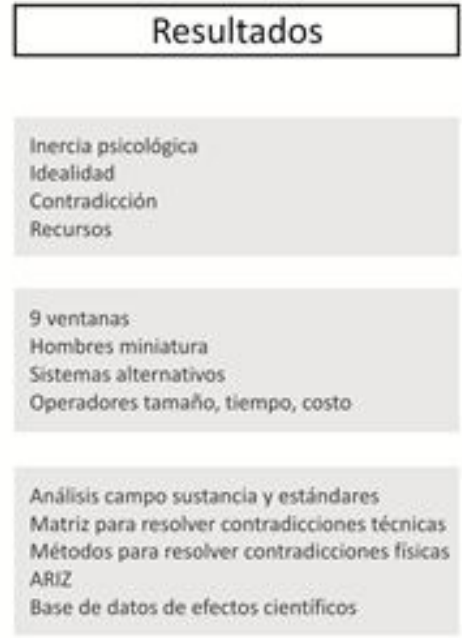

Figura 1. Clasificación del trabajo de Altshuller en torno a TRIZ. Basado en Cavallucci (2002) 


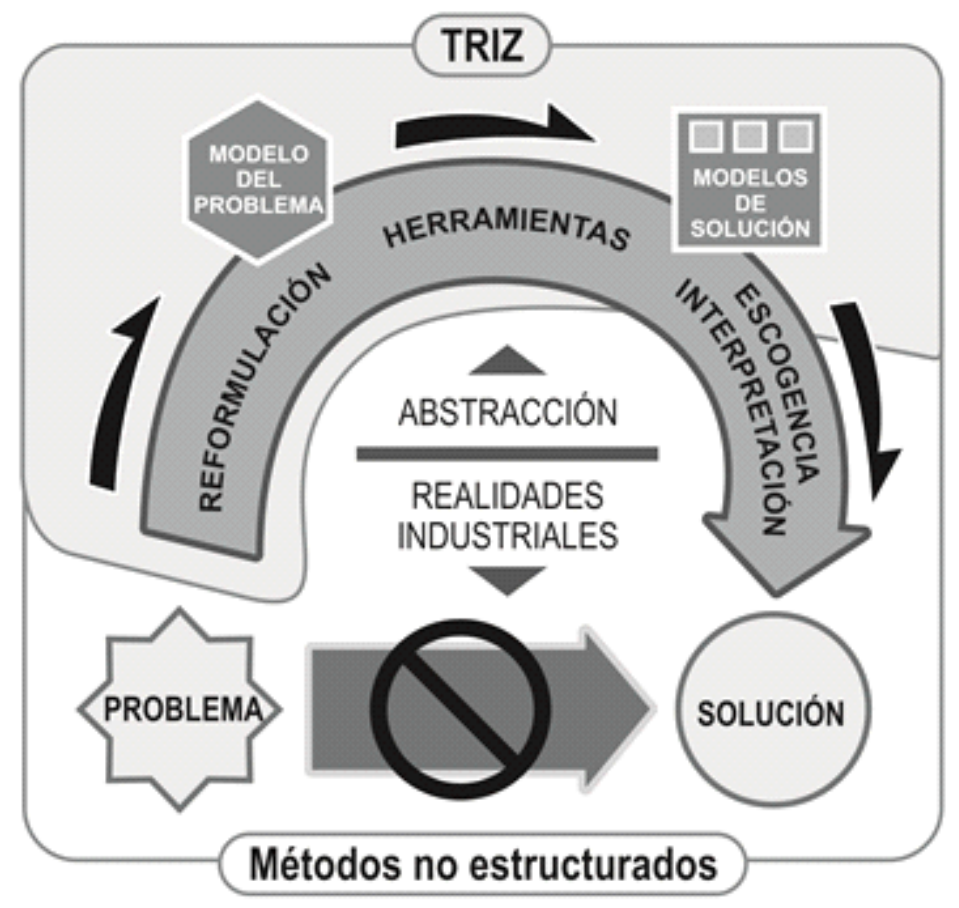

Figura 2. Característica de la metodología TRIZ para la solución de un problema. Basado en Cavallucci (2002)

la energía del exterior para realizar dicha función sobre un objeto (Savransky 2001). El sistema se modela con cuatro componentes: motor, transmisión, herramienta y control como se muestra en la Figura 3. El motor es la parte del sistema que convierte la energía del exterior en otra, para asegurar la función principal; la transmisión conduce la energía desde el motor hacia la herramienta; la herramienta, o unidad de trabajo, asegura el contacto entre el sistema y el objeto donde se realiza la función principal; y el control, reacciona a los cambios del sistema adaptándose automáticamente a su forma, estructura o salida (Cavallucci \& Weill 2001).

Para TRIZ, los ST presentan un desarrollo lógico evolutivo, cuyo propósito es alcanzar la idealidad, a través del mejoramiento de su desempeño en el tiempo. El estado de un producto (Sistema Técnico) en su proceso evolutivo puede determinarse con el nivel de cumplimiento de las Leyes de Evolución propuestas por Altshuller (Cavallucci 2002), de esta forma, evaluar las leyes en un punto específico del desarrollo de un ST permite identificar oportunidades de innovación futuras. En la actualidad, las Leyes de
Evolución son concebidas como tendencias de los ST y se sugiere un número superior a las ocho originales (Moehrle 2005).

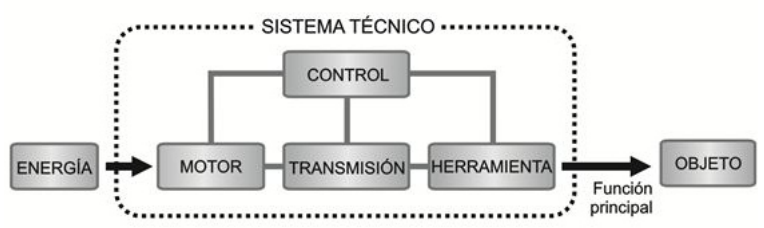

Figura 3. Partes del Sistema Técnico. Basado en Cavallucci $\& \operatorname{Weill}(2001)$

\subsubsection{Clasificación de las Leyes de Evolución}

\section{Leyes estáticas}

Son aquellas que permiten evaluar la completitud estructural y funcional del sistema.

Ley 1. Completitud de las partes: establece que un sistema llevará a cabo adecuadamente su función principal cuando presenta sus cuatro partes (motor, transmisión, herramienta y control). 
Ley 2. Flujo de energía: establece que debe existir un flujo libre y eficiente de la energía a través de los cuatro elementos del sistema. Las pérdidas pueden estimarse con la diferencia entre la energía de entrada y la de salida, entregada a la herramienta.

Ley 3. Coordinación del ritmo de las partes: establece que un sistema debe tener sincronización en su frecuencia, vibración y periodicidad de todas sus partes. Una diferencia en la coordinación entre los elementos puede causar pérdidas, y otros efectos nocivos, en todo el sistema.

\section{Leyes cinemáticas}

Son aquellas que permiten observar el sistema en una visión espacio-temporal desde el pasado para analizar su evolución tecnológica.

Ley 4. Idealidad: establece que todo ST tiende hacia la perfección. El sistema ideal es aquel que lleva a cabo su función principal sin consumo de materia ni energía y con el mínimo costo.

Ley 5. Desarrollo desigual de las partes: establece que cuanto más desigual sea el desarrollo de las partes, el sistema tiende a ser más complejo y menos evolucionado. Estas desigualdades llevan a contradicciones técnicas y físicas, que pueden ser la base de la evolución futura.

Ley 6. Transición al supersistema: establece que cuando un sistema ha alcanzado un alto nivel de desarrollo puede unirse a un supersistema y llegar a ser parte de él.

\section{Leyes dinámicas}

Son aquellas que establecen una proyección del sistema en el futuro. Están constituidas por dos leyes excluyentes, es decir, un sistema sólo puede seguir una u otra ley.

Ley 7. Transición de macro nivel a micro nivel: establece la existencia de una tendencia del sistema hacia la miniaturización de sus partes, tales como micro-instrumentos, micro-electrónica yotras.
Ley 8. Aumento de la dinamicidad y controlabilidad: establece que los sistemas rígidos deben orientarse hacia la segmentación, mientras aumentan su control, a través de las transiciones de campos (mecánicos, eléctricos, magnéticos y electromagnéticos).

La determinación del estado de evolución de un producto, con base en el cumplimiento de las Leyes de Evolución, se realiza a través de una escala relativa y no absoluta. Cavallucci \& Weill (2002) sugieren una escala cualitativa de valoración de cero a tres de acuerdo al grado de cumplimiento de la ley; de esta forma, se designa cero (0), si la ley no se cumple; uno (1), si se cumple en algunas situaciones; dos (2), si se verifica en muchas situaciones; y tres (3), si la ley se cumple completamente. En un diagrama de radar se visualiza el estado evolutivo del Sistema Técnico, de acuerdo a las asignaciones de cumplimiento para cada ley.

Las oportunidades de innovación en los ST requieren analizar el estado del producto en cada una de las leyes y de acuerdo a su estado, se pueden generar propuestas de innovación para el mejoramiento del producto (Cavallucci 2002)

\section{Método para identificar oportunidades de innovación en productos de apoyo}

Según AENOR (2007), un producto de apoyo es cualquier dispositivo, instrumento, software, tecnología o equipo, fabricado especialmente o disponible en el mercado para prevenir, compensar, controlar, mitigar o neutralizar la discapacidad de un individuo. La norma establece una clasificación en tres niveles jerárquicos (clase, subclase y divisiones), según la función que ofrecen; la Figura 4 muestra un mapa conceptual de la norma donde se detalla la clase 12 , correspondiente a los productos de apoyo para la movilidad personal.

Para el análisis de la Evolución de los Sistemas Técnicos, aplicados a productos de apoyo para la movilidad personal, se sugieren un conjunto de cinco pasos:

Seleccionar las subclases a analizar, dentro de la norma UNE-EN ISO 9999 (2007) de acuerdo a la necesidad identificada en la población en 
situación de discapacidad. Se sugiere un trabajo interdisciplinar del área de salud, el diseño y la ingeniería, puesto que los productos de apoyo deben atender a necesidades que van más allá de lo técnico (Gonzalez-Cruz et al 2009).

Revisar, en el ámbito mundial, los productos comerciales más recientes pertenecientes a las subclases elegidas, y seleccionar un ejemplar de referencia para cada una. Para el proceso de selección del producto de referencia debe priorizarse el aspecto asociado con la necesidad identificada, a su vez debe realizarse un proceso de vigilancia tecnológica con los fabricantes de dichos productos. Un conjunto de criterios para la selección son: propiedades relevantes del producto frente a la necesidad, las ventajas y el cumplimiento de normativas internacionales.

Modelar los productos de referencia como Sistema Técnico. Se debe analizar los productos de apoyo como un sistema integral hombremáquina que busca realizar una función principal acorde a la necesidad identificada. Los elementos del sistema técnico son el motor, la transmisión, la herramienta y el control que deben ser abordados de manera conjunta hombre-máquina.

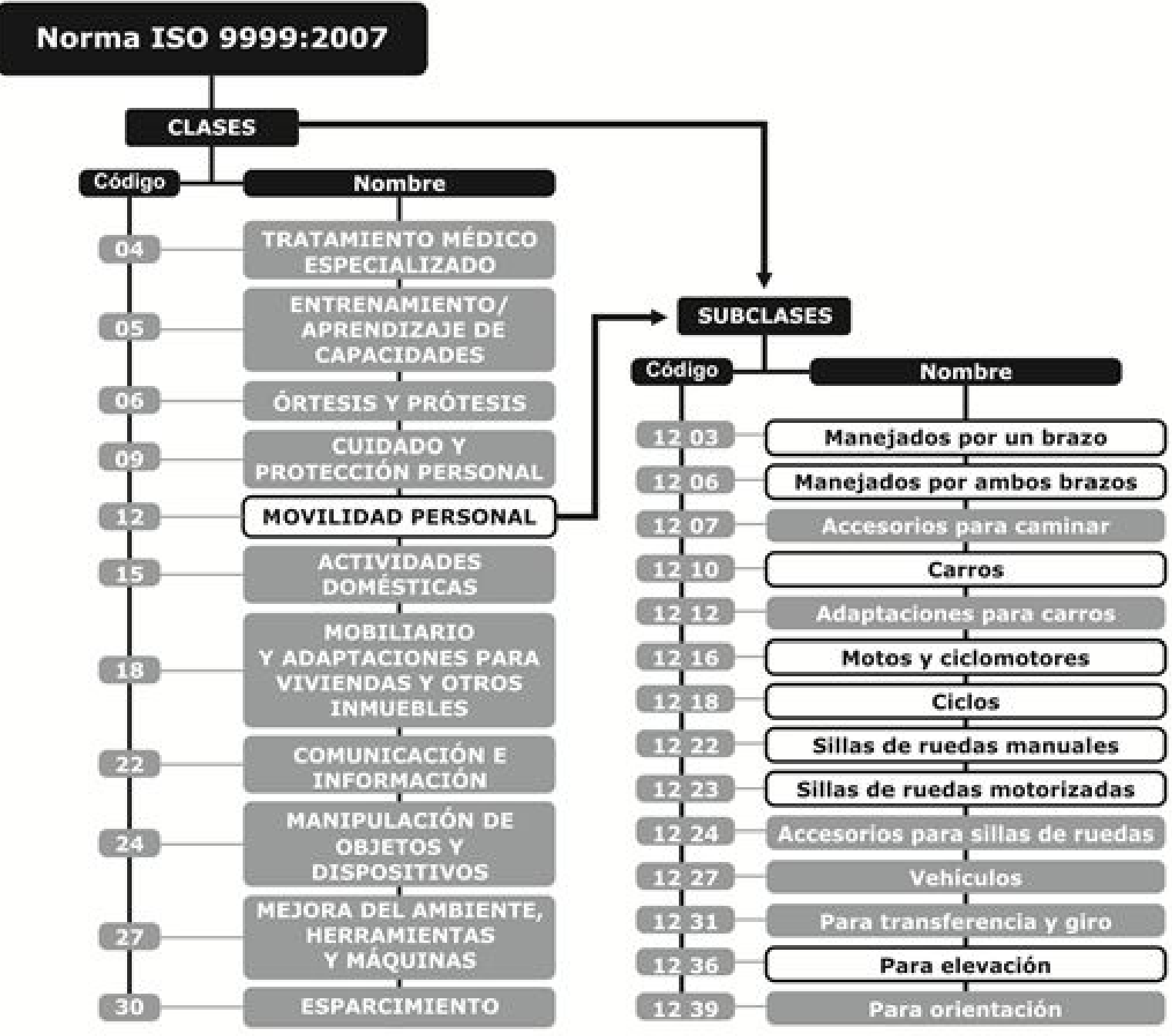

ftems de estudio

Figura 4. Mapa conceptual de la norma UNE-EN ISO 9999. Datos: AENOR (2007) 
Analizar la Evolución de los Sistemas Técnicos para cada producto de referencia. Consiste en evaluar de manera cualitativa el cumplimiento de las leyes de evolución de los sistemas técnicos propuestas en la teoría TRIZ (Cavallucci 2002).

Identificar las oportunidades de innovación en las subclases de análisis, con base en el estado de evolución actual de los productos de referencia. A partir de unos diagramas de radar, en aquellas leyes donde el cumplimiento es bajo, se debe identificar la oportunidad de innovación que se traduce en mejoras del sistema técnico con base en las deficiencias encontradas.

\section{Resultados y discusión}

\subsection{Selección de subclases a analizar}

El proyecto de investigación abordó el problema de la inclusión social. A partir del problema se realizó un análisis interdisciplinar multicriterio para detectar que tipo de familia de productos debería ser diseñada para favorecer la inclusión social (González-Cruz et al 2009). De la clase 12 de productos de apoyo a la movilidad, se seleccionaron ocho subclases para el análisis de evolución, las seis subclases restantes se descartaron por ser accesorios de los productos. Las subclases que se consideraron, junto a su número de clasificación, fueron las siguientes: productos de asistencia manipulados por un brazo o bastones (12 03), productos de asistencia manipulados por dos brazos o andadores (12 06), ciclos (12 18), sillas de ruedas de propulsión manual (12 22), productos de asistencia para traslados (12 36), motocicletas y ciclomotores (12 $16)$, carros (12 10) y sillas de ruedas motorizadas (1223).

\subsection{Revisión de productos comerciales a nivel mundial y selección de ejemplares representativos}

Se revisaron las especificaciones técnicas de productos disponibles actualmente en el mercado, entre las empresas fabricantes y distribuidoras a nivel mundial. Se registraron 140 productos en total, los cuales están asociados a 45 empresas ubicadas en 11 países como se muestra en la Tabla 1.

El producto con mejores prestaciones, mediante la comparación de sus características técnicas, se seleccionó como referencia para el análisis de evolución de cada subclase (véase Figura 5). Por ejemplo, para la subclase sillas de ruedas de propulsión manual (12 22) se eligió una silla con palancas. Esta selección obedece a un proceso de análisis comparativo con la silla de propulsión por aro con base en los criterios de consumo de energía y ventaja mecánica. A su vez, por el cumplimiento de estándares internacionales.

El proceso para el análisis comparativo fue el siguiente: el consumo energético para los dos productos se evaluó a partir de la relación de potencias de acuerdo a la Figura 6, donde es

Tabla 1. Resultados de la revisión de productos de apoyo a la movilidad, según empresas productoras. ${ }^{\mathrm{c}} \mathrm{y}{ }^{\mathrm{d}}$ Valores en frecuencia. Clasificación norma UNE-EN ISO9999

\begin{tabular}{|c|c|c|c|}
\hline \multicolumn{4}{|c|}{ Productos } \\
\hline Código & Nombre & Evaluados $^{\mathrm{c}}$ & Empresas $^{\mathrm{d}}$ \\
\hline 1203 & Caminador de un brazo & 14 & 6 \\
\hline 1206 & Caminador de ambos brazos & 23 & 12 \\
\hline 1210 & Coche/Automóvil & 4 & 2 \\
\hline 1216 & Ciclomotor & 15 & 8 \\
\hline 1218 & Ciclos & 17 & 7 \\
\hline 1222 & Sillas de ruedas manual & 16 & 8 \\
\hline 1223 & Sillas de ruedas eléctrica & 27 & 5 \\
\hline 1236 & Elevador/Grúa & 24 & 13 \\
\hline
\end{tabular}




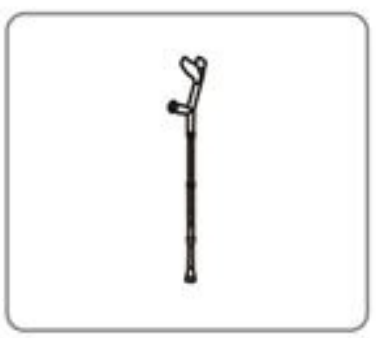

Caminador de un brazo (12 03)

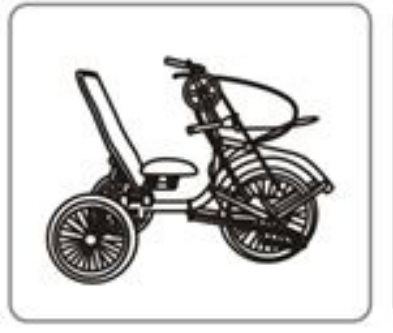

Ciclo

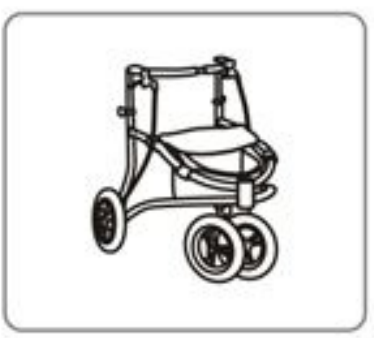

Caminador de ambos brazos (1206)

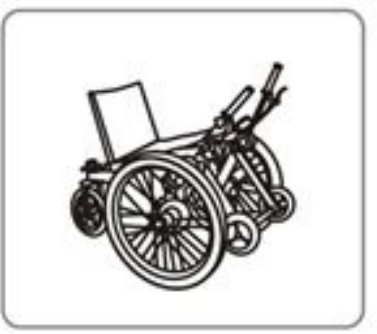

Silla de ruedas manual

(12 22)

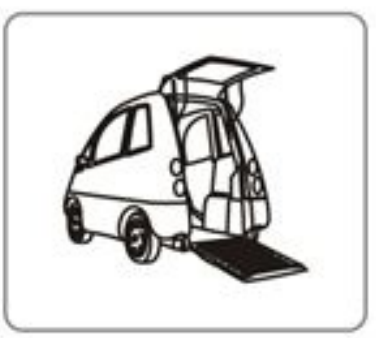

Coche/Automóril

(12 10)

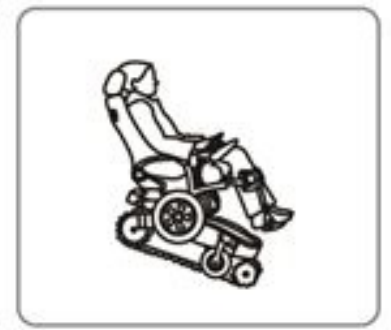

Silla de ruedas eléctrica

(12 23)

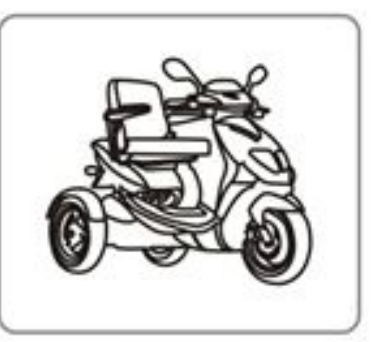

Ciclomotor

(12 16)

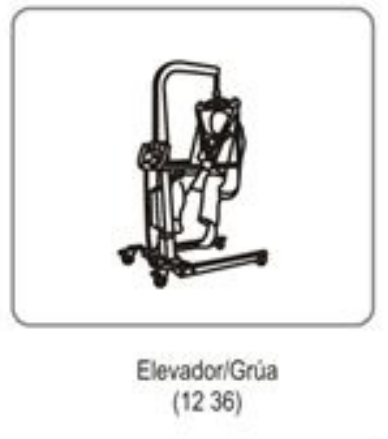

En paréntesis codigo de la norma ISO 9999:2007

Figura 5. Productos de referencia para el análisis de Evolución de los Sistemas Técnicos

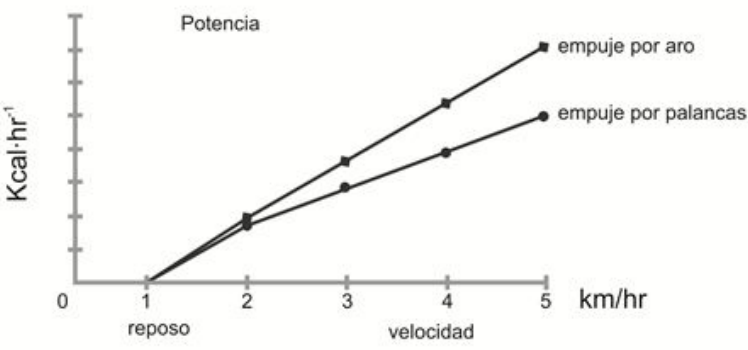

Figura 6. Relación de potencias entre la silla de empuje por aro y la silla de empuje por palancas (Engel \& Seeijger, 1986)

evidente que la silla con palancas presenta menor consumo energético que la de propulsión por aro (Engel \& Seeijger 1986). Frente a la ventaja mecánica, se comparó el torque de salida de ambas sillas el cual depende de la fuerza que proporcione la persona y la distancia al eje de la rueda de acuerdo al punto de aplicación de la fuerza, conforme Ec. (1)

$$
\tau=\mathrm{F} \cdot \mathrm{d}
$$

Donde $\tau$ es el torque que suple la persona, $\mathrm{F}$ la fuerza proporcionada por la persona y d la distancia desde el apoyo al punto de aplicación de la fuerza.

La Figura 7 ilustra este tipo de fuerzas para ambas sillas en un instante de tiempo dado; allí se observa que $\mathrm{r}_{2}>\mathrm{r}_{1}$ y $\mathrm{F}_{2}>\mathrm{F}_{1}$, con lo cual se evidencia que el torque de salida de la silla con palancas es mayor en comparación al de la silla con aro y ofrece una ventaja mecánica superior, debido a su brazo de fuerza.

\subsection{Modelado de los productos de referencia como sistema técnico}

Los productos de apoyo son extensiones de la persona que buscan superar una limitación. Cuando una persona supera la limitación, el producto de apoyo pierde su utilidad para ella. Desde este punto de vista, los productos de apoyo complementan a la persona para suplir su limitación. Con base en esto, el análisis de este 

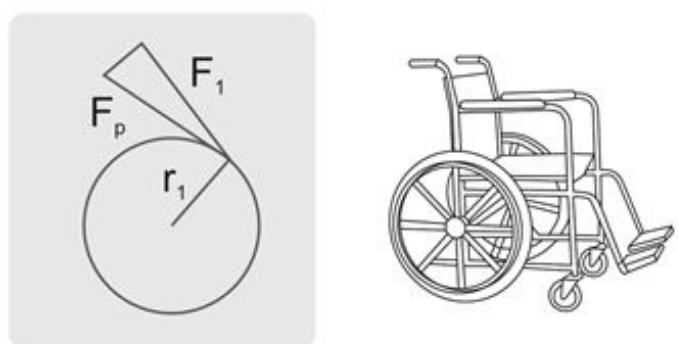

a) Fuerza ejercida por la persona en la silla con aro de empuje
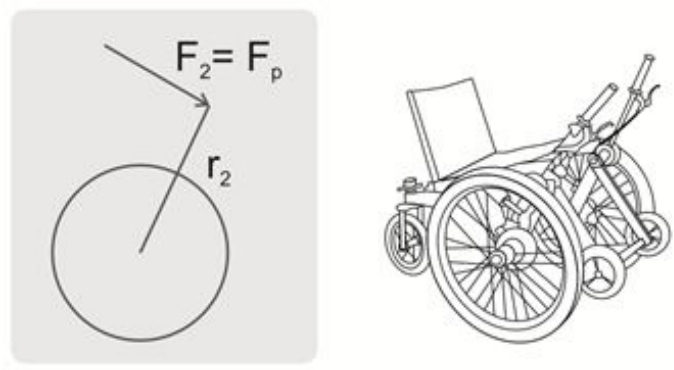

b) Fuerza ejercida por la persona en la silla con palanca

$F_{r}$ : fuerza aplicada por la persona

$F_{\text {. componente tangencial al aro de empuje }}$

$F$, fuerza aplicada en la palanca

Figura 7. Torques de las sillas propulsadas por aro y por palancas

tipo de productos requiere de un tratamiento especial para ser representados en forma de Sistema Técnico (ST) puesto que se consideran como sistemas híbridos hombre-máquina, donde la operación de la máquina (producto) está subordinada a la acción del hombre (usuario). Por ejemplo, para el caso de los productos de apoyo a la movilidad, la Función Principal es desplazar a la persona debido a la limitación de las extremidades inferiores, lo cual requiere de un producto que supla dicha limitación. Así, la persona es la base del ST puesto que forma parte integral de ella para realizar esa función principal. De esta forma, su sistema muscular de miembros superiores actúa como motor, su estructura ósea como transmisión o herramienta, y su sistema nervioso como control central del sistema; de este modo, el producto de apoyo compensa las funciones fisiológicas del usuario, limitadas por la discapacidad. Se analizaron, bajo esta óptica de sistema híbrido, varios tipos de productos con base en la clase 12 de la norma ISO9999 como muestra en la Tabla 2.
Como ejemplo del proceso de modelado, se describen las partes del ST para la subclase 12 22, cuyo producto de referencia es una silla de ruedas de propulsión con palancas (véase Figura 8). La función principal, dentro del lenguaje TRIZ, es desplazar a la persona y existen otras funciones asociadas a la función principal como son la de portar al usuario, proveer confort y seguridad.

Motor: los músculos de los miembros superiores del usuario; proporcionan la fuerza a la transmisión, junto con la cual facilitan la movilidad del sistema.

Transmisión: los tendones, huesos y articulaciones de los miembros superiores del usuario y además, la palanca y trasmisión interna de la silla, la cual consiste en un trinquete que permite ejercer fuerza en una sola dirección.

Control: el sistema nervioso del usuario y los elementos físicos de la silla que permiten las funciones de avance, giro y frenado. El avance se realiza a través del movimiento de ambas palancas, el giro por el control del movimiento independiente de las ruedas y el frenado por suspensión del avance, mediante el accionamiento de un freno manual.

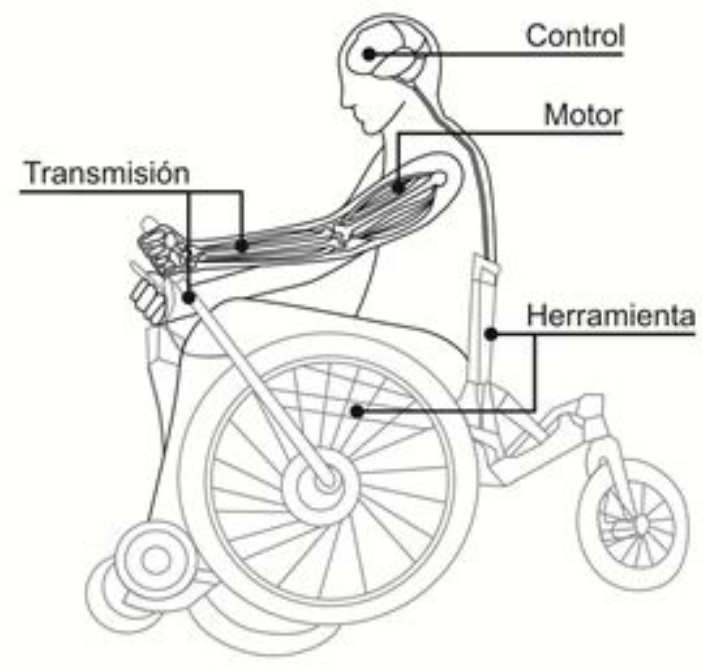

Figura 8. Partes del Sistema Técnico para la subclase 1222 
Tabla 2. Modelado como Sistemas Técnicos de los productos de referencia para las subclases de estudio ${ }^{\mathrm{a}} \mathrm{y}{ }^{\mathrm{b}}$ Según clasificación norma UNE-EN ISO 9999

\begin{tabular}{|c|c|c|}
\hline \multicolumn{3}{|c|}{ Productos } \\
\hline Código $^{\mathrm{a}}$ & Nombre ${ }^{\mathrm{b}}$ & Descripción \\
\hline \multirow[t]{6}{*}{1203} & Caminador de un brazo & \\
\hline & Función & Soportar y estabilizar \\
\hline & Motor & Músculos activos de las extremidades inferiores, brazos y torso \\
\hline & Transmisión & Tendones y articulaciones de brazos y piernas presentes \\
\hline & Herramienta & Extremidades inferiores y bastón \\
\hline & Control & Sistema nervioso \\
\hline \multirow[t]{6}{*}{1206} & Caminador de ambos brazos & \\
\hline & Función & Soportar y estabilizar \\
\hline & Motor & Músculos activos de las extremidades inferiores, cadera, brazos y torso \\
\hline & Transmisión & Tendones y articulaciones de brazos y piernas presentes \\
\hline & Herramienta & Extremidades inferiores y caminador \\
\hline & Control & Sistema nervioso \\
\hline \multirow[t]{6}{*}{1210} & Coche/Automóvil & \\
\hline & Función & Desplazar y Soportar \\
\hline & Motor & Motor de combustible fósil \\
\hline & Transmisión & Mecanismos de transmisión del coche (embrague, caja de cambios) \\
\hline & Herramienta & Cabina del auto \\
\hline & Control & Palancas de cambios, padales de embrague, aceleración y frenado \\
\hline \multirow[t]{6}{*}{1216} & Ciclomotor & \\
\hline & Función & Desplazar, soportar y estabilizar \\
\hline & Motor & Motor de combustible fósil \\
\hline & Transmisión & Mecanismos de transmisión del ciclomotor \\
\hline & Herramienta & Silla del ciclomotor \\
\hline & Control & Acelerador, freno, embrague y dirección \\
\hline \multirow[t]{6}{*}{1218} & Ciclo & \\
\hline & Función & Desplazar, soportar y estabilizar \\
\hline & Motor & Músculos activos de las extremidades superiores \\
\hline & Transmisión & Tendones y articulaciones de extremidades superiores e inferiores \\
\hline & Herramienta & Extremidades inferiores, superiores y ciclo \\
\hline & Control & Sistema nervioso \\
\hline \multirow[t]{6}{*}{1222} & Silla de ruedas manual & \\
\hline & Función & Desplazar, soportar y estabilizar \\
\hline & Motor & Músculos de los miembros superiores \\
\hline & Transmisión & $\begin{array}{l}\text { Tendones, huesos y articulaciones de brazos y mecanismos de transmisión de la } \\
\text { silla }\end{array}$ \\
\hline & Herramienta & Silla, espaldar y reposabrazos \\
\hline & Control & Sistema nervioso \\
\hline \multirow[t]{6}{*}{1223} & Silla de ruedas eléctrica & \\
\hline & Función & Desplazar, soportar y estabilizar \\
\hline & Motor & Motor eléctrico \\
\hline & Transmisión & Mecanismos de transmisión de la silla de ruedas motorizada \\
\hline & Herramienta & Silla, espaldar y reposabrazos \\
\hline & Control & Controles de dirección y avance \\
\hline \multirow[t]{5}{*}{1236} & Elevador /Grúa & \\
\hline & Función & Desplazar, soportar y estabilizar \\
\hline & Motor & Motor eléctrico \\
\hline & Transmisión & Estructuras móviles de la grúa \\
\hline & Herramienta & Arneses \\
\hline
\end{tabular}

Herramienta: la rueda junto con el asiento, espaldar y demás elementos físicos de la silla que soportan al usuario mientras se moviliza. La razón para que la rueda sea la herramienta del sistema técnico es porque en la definición de sistema técnico, la herramienta es el elemento que entra en contacto directo con el objeto (suelo) para realizar la función principal (desplazar). 


\subsection{Análisis del cumplimiento de las leyes de evolución de los sistemas técnicos para los productos de referencia}

Para este análisis es necesario seleccionar un Sistema Técnico ideal hacia el cual, según el concepto de Evolución, debe tender todo ST. Dada la característica híbrida del sistema en consideración, la idealidad se definió como la persona caminante sin deficiencias en movilidad.

Se analizó el grado de cumplimiento de las Leyes de Evolución para cada producto de referencia y, según la escala establecida, se asignó un valor. La Figura 9 muestra los diagramas de radar resultantes.

A manera de ejemplo, se muestra el proceso de evaluación de las leyes para la silla de ruedas de propulsión con palancas:

Ley 1. Completitud del sistema: la silla presenta las cuatro partes del ST, sin embargo, el motor (músculos de los miembros superiores del usuario) tiene una capacidad de potencia inferior al ideal de una persona caminante. Esto se deriva al comparar el torque pico en excéntrico de la pierna y del brazo como se muestra en la Tabla 3.
Tabla 3. Comparación entre torques pico de brazos y piernas, según edad.

Lynch et al., (1999)

\begin{tabular}{ccc}
\hline \multicolumn{3}{c}{ Torque pico en excéntrico $(\mathrm{N}-\mathrm{m})$} \\
\hline Edad (años) & brazo & pierna \\
\hline $20-29$ & 217 & 429 \\
$30-39$ & 227 & 401 \\
$40-49$ & 217 & 409 \\
$50-59$ & 203 & 383 \\
$60-69$ & 175 & 337 \\
$70-79$ & 162 & 289 \\
más de 80 & 139 & 257 \\
\hline
\end{tabular}

Este resultado es un indicador de insuficiencia en la completitud del sistema, con lo cual se asignó grado dos (2) de cumplimiento a esta ley.

Ley 2. Flujo de energía: la propulsión por palancas presenta una eficiencia del $10.2 \%$, la cual no equipara a la de una persona caminante que está dentro del 25\% (Van der Woude 1997). Esta condición es un indicador de pérdidas de energía del sistema por lo cual se asignó un grado dos (2) de cumplimiento.
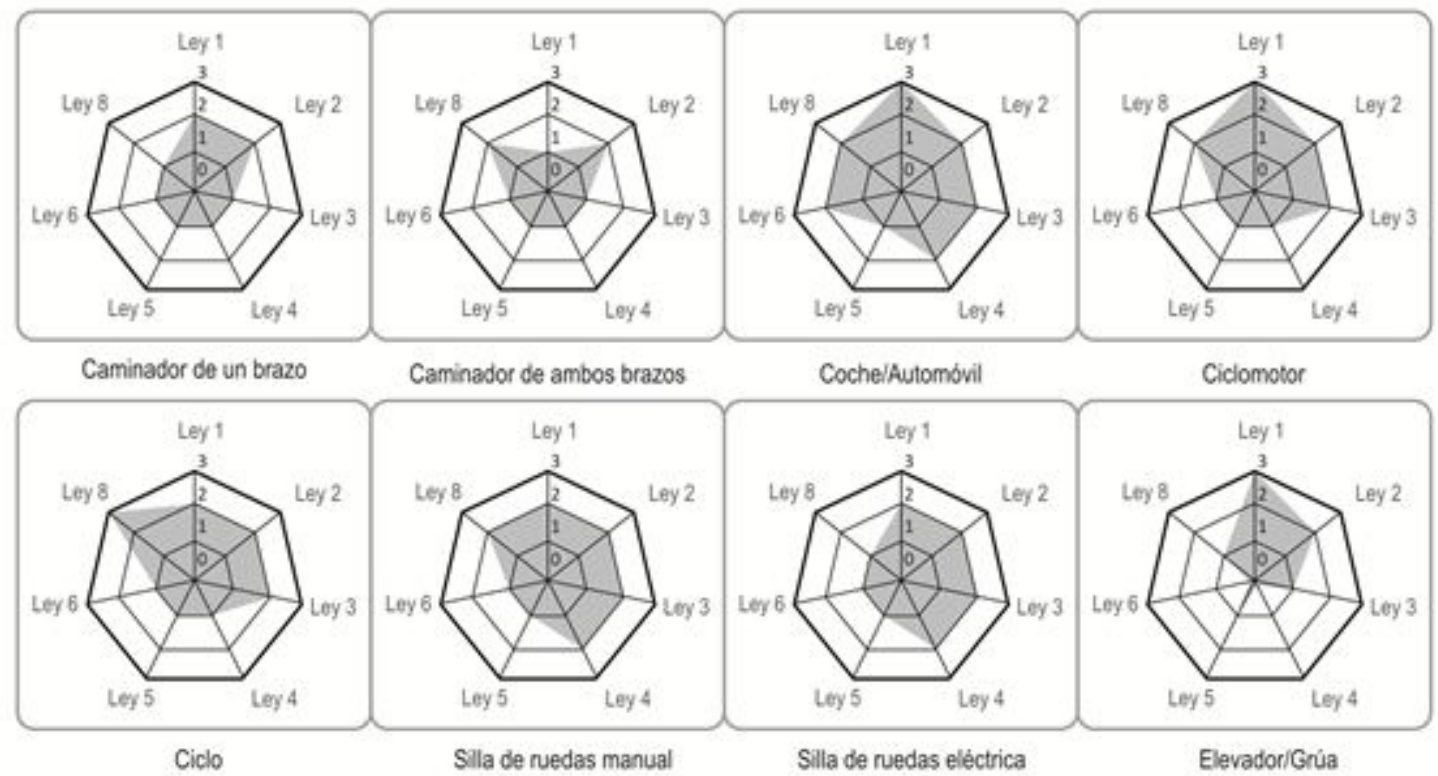

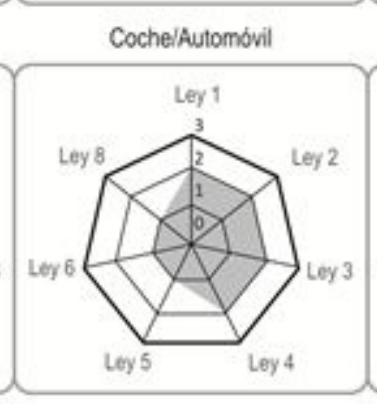

Silla de ruedas eléctrica

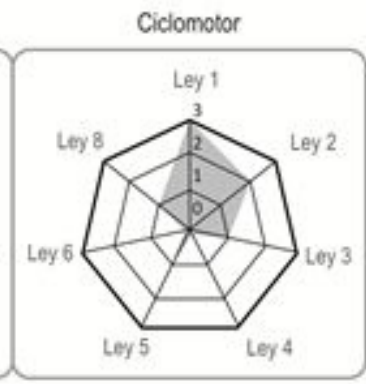

Elevador/Grúa

Figura 9. Cumplimiento de las Leyes de Evolución de los Sistemas Técnicos para los productos de referencia 
Ley 3. Coordinación del ritmo entre las partes: la propulsión por palancas se aproxima a la marcha de un caminante en el movimiento de las extremidades superiores, puesto que los impulsos están desfasados para proporcionar un ritmo natural en el movimiento que es favorable a la coordinación de las partes. Sin embargo, el sistema presenta una desarmonía en el ritmo de las partes debido a las vibraciones generadas por la ausencia de un mecanismo de suspensión; por lo tanto, se estableció grado dos (2) de cumplimiento de esta ley.

Ley 4. Aumento en la idealidad: se busca que los productos de apoyo propicien la autonomía personal en la realización de actividades que favorezca la inclusión social. Desde este aspecto, con base en la función principal de desplazar, se consideraron aspectos como el área requerida para la movilidad, la accesibilidad a las edificaciones y a los medios de transporte. En cuanto a la autonomía, la silla con palancas posibilita el tránsito sobre terrenos no uniformes y el desplazamiento por terrenos inclinados sin el riesgo de devolverse; con respecto al área requerida para la movilidad no hay desarrollo; frente a la accesibilidad al medio, la silla presenta limitaciones en el uso de baños y sistemas de transporte. A esta ley se asignó grado dos (2) de cumplimiento.

Ley 5. Desarrollo desigual de las partes: la silla con palancas, en la parte asociada con la herramienta del sistema, cuenta con estructuras antivuelco y de adaptabilidad a terrenos no uniformes. Sin embargo, el motor, la trasmisión y el control del Sistema Técnico requieren de mayores desarrollos que favorezcan el confort, la controlabilidad y la eficiencia del sistema para evolucionar en esta ley, por lo tanto, se estableció cumplimiento en grado uno (1). Los desarrollos históricos, como se muestra en la Figura 10, dan cuenta de la baja evolución en el motor, transmisión y control del sistema.

Ley 6. Transición al supersistema: La idealidad de un producto de apoyo es que permita al usuario vencer la limitación para que se integre a la sociedad (supersistema) de igual forma que aquel que no tiene limitación. Desde esta óptica, con la base en la función principal, la silla con palancas

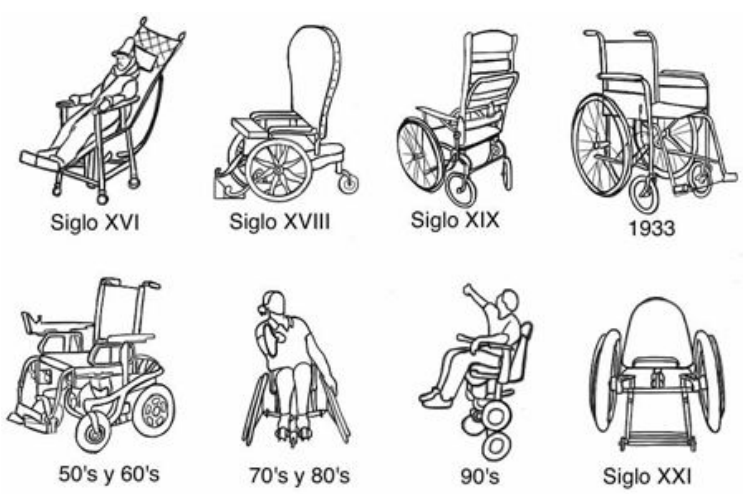

Figura 10 Evolución de los productos de apoyo para movilidad

ayuda al desplazamiento por aceras con bordillos bajos y terrenos no pavimentados, sin embargo, permanecen algunas actividades sin solución como subir y bajar escaleras de edificios, acceder a los medios de transporte, cruzar por puertas angostas, y realizar traslado, para que se integre efectivamente al entorno físico; con lo cual se estipuló grado uno (1) de cumplimiento.

Ley 8. Aumento de la dinamicidad y controlabilidad: la transmisión de la silla con palancas permite multiplicar el efecto del empuje a través de movimientos naturales de las extremidades superiores, lo cual constituye un indicador del aumento en la dinamicidad del sistema; sin embargo, la controlabilidad permanece estática, pues el control depende únicamente del usuario y no existen mecanismos de seguridad para situaciones de riesgo. Por lo tanto, se asignó grado dos (2) de cumplimiento.

\subsection{Identificación de oportunidades de innovación en las subclases de análisis de los productos de apoyo a la movilidad}

Se identificó un conjunto de posibilidades de innovación para las subclases analizadas a partir del estado de evolución de cada producto. La Tabla 4 presenta las oportunidades de innovación más relevantes para estos productos. A manera de ejemplo, se muestra en detalle el resultado del proceso de análisis para la subclase 1222 :

Ley 1. Completitud del sistema: debe proveerse una fuente de potencia auxiliar para compensar las 
desventajas mecánicas de las extremidades superiores comparada con las inferiores.

Ley 2. Flujo de energía: deben buscarse otras formas mecánicas de tracción que permitan aumentar la eficiencia en la propulsión del sistema.

Ley 3. Coordinación del ritmo entre las partes: debe dotarse al sistema con un mecanismo de suspensión para protección y comodidad del usuario.

Ley 4. Aumento en la idealidad: debe disminuirse el área del sistema para proveer mayores posibilidades de acceso.

Ley 5. Desarrollo desigual de las partes: se requiere mayor trabajo en la interacción del usuario-máquina que favorezca la ergonomía, el uso eficiente de energía y el control.
Ley 6. Transición al supersistema: se debe proveer al sistema de nuevas funciones que permitan el acceso a los servicios de transporte y a las actividades de subir y bajar escaleras de edificaciones.

Ley 8. Aumento de la dinamicidad y controlabilidad: se requiere de un sistema de control para situaciones de riesgo, como bajar pendientes, así como también de un sistema de ayuda al desplazamiento cuando se requiera realizar la propulsión con un solo brazo.

\section{Conclusiones}

Se ha propuesto una estrategia para identificar oportunidades de innovación, que es la aplicación de conocimiento en la solución de problemas, para los productos de apoyo a la movilidad con base en el análisis del cumplimiento de las leyes de

Tabla 4. Oportunidades de innovación para los productos de apoyo a la movilidad. clasificación norma UNE-ENISO 9999

\begin{tabular}{|c|c|c|}
\hline \multicolumn{3}{|c|}{ Producto } \\
\hline Código & Nombre & Oportunidades de innovación \\
\hline 1203 & \multirow{3}{*}{ Caminador de un brazo } & Mejorar la disipación del impacto y la unión del usuario con el bastón. \\
\hline & & $\begin{array}{l}\text { Buscar alternativas para liberar al usuario del uso de las extremidades } \\
\text { superiores conservando el patrón de marcha. }\end{array}$ \\
\hline \multirow{4}{*}{1206} & & Optimizar el sistema para uso en diferentes terrenos. \\
\hline & \multirow{3}{*}{ Caminador de ambos brazos } & $\begin{array}{l}\text { Optimizar los rodamientos para reducir la fricción y completar la demanda } \\
\text { energética que se requiere. }\end{array}$ \\
\hline & & Mejorar la administración y control del sistema. \\
\hline & & Disminuir el área del sistema sin detrimento de la función. \\
\hline \multirow[t]{3}{*}{1210} & \multirow{3}{*}{ Coche/Automóvil } & $\begin{array}{l}\text { Proveer características que aumenten la seguridad y la ergonomía del } \\
\text { conductor. }\end{array}$ \\
\hline & & Optimizar el uso de energía. \\
\hline & & Mejorar acceso al vehículo. \\
\hline \multirow[t]{2}{*}{1216} & \multirow[b]{2}{*}{ Ciclomotor } & Buscar de alternativas para accesibilidad del usuario al sistema. \\
\hline & & $\begin{array}{l}\text { Optimizar mecanismos de transmisión, administración y control. } \\
\text { Buscar alternativas de compensación de carga. }\end{array}$ \\
\hline \multirow[t]{2}{*}{1218} & \multirow[b]{2}{*}{ Ciclo } & Búsqueda de mecanismos alternos que permitan, a partir de movimientos \\
\hline & & $\begin{array}{l}\text { naturales, impulsar el sistema. } \\
\text { Optimizar el flujo de energía del sistema en procesos de aceleración y } \\
\text { desaceleración. }\end{array}$ \\
\hline \multirow[t]{2}{*}{1222} & \multirow[b]{2}{*}{ Silla de ruedas manual } & Aumentar la controlabilidad del sistema. \\
\hline & & $\begin{array}{l}\text { Buscar sistemas de asistencia para desplazamiento en condiciones de alto } \\
\text { esfuerzo. } \\
\text { Reducir el área del sistema. }\end{array}$ \\
\hline \multirow[t]{2}{*}{1223} & \multirow{2}{*}{ Silla de ruedas eléctrica } & $\begin{array}{l}\text { Optimizar la transmisión del sistema que permita reducir el consumo de } \\
\text { energía. }\end{array}$ \\
\hline & & $\begin{array}{l}\text { Mejorar la transición en el cambio de funciones en el proceso de adaptación } \\
\text { a nuevas condiciones del medio. }\end{array}$ \\
\hline 1236 & Elevador /Grúa & $\begin{array}{l}\text { Proveer opciones de interacción con otros subsistemas para la realización } \\
\text { de otras actividades por el usuario }\end{array}$ \\
\hline
\end{tabular}


evolución de los sistemas técnicos de la teoría desolución de problemas inventivos TRIZ.

Se ha sugerido un modelo de caracterización de los Sistemas Técnicos de ayuda a la movilidad a partir de una integración de la máquina y la persona como un solo sistema híbrido hombre-máquina, compuesto por el motor, transmisión, herramienta y control.

Se ha realizado un análisis multidisciplinar de los productos de apoyo a la movilidad desde una mirada sistémica y evolutiva, que ha permitido ampliar la visión del problema y garantizar el tránsito del producto a niveles óptimos de funcionamiento.

Se ha encontrado que en general los productos de apoyo tienen deficiencias en cuanto a la administración y control del sistema técnico (hombre-máquina) lo cual no ayuda en la superación de la limitación que es la idealidad del sistema. De esta forma, las oportunidades de innovación ayudan a enfocar el trabajo investigativo, siendo el reto más importante el de vencer la limitación a partir de la restauración de la función de control que está asociada con el sistema nervioso de la persona.

El bajo nivel de cumplimiento de algunas de las Leyes de Evolución de los Sistemas Técnicos analizados, parece indicar que el diseño de estos productos ha surgido de la adaptación de otros elementos pensados para otras necesidades y por lo tanto se requiere del uso de la aproximación hombre-máquina para optimizar este tipo de productos.

\section{Agradecimientos}

Esta investigación se desarrolló en el proyecto de investigación: "Diseño Conceptual interdisciplinario, a partir de un Modelo Ampliado del Diseño Axiomático, de Ayudas Técnicas y Tecnológicas para Movilidad Personal que favorezcan la Inclusión Social de personas en situación de discapacidad", Proyecto ANDAR, financiado por el Departamento Administrativo de Ciencia, Tecnología e Innovación COLCIENCIAS. Cód. 1251-452-21059. CT. 6522008.

\section{Referencias bibliográficas}

AENOR (Asociación Española de Normalización y Certificación). (2007). Productos de apoyo para personas en situación de discapacidad: Clasificación y terminología (UNE-EN ISO9999).

Aguilar, J. A., Prada, M., Gómez, E. \& González, M. (2008). Analysis and identification of research opportunities in product design using the multidimensional project model. In Proceedings of the International Conference DESIGN 2008, Duvrovnik, Croatia, p.89-96.

Cavallucci, D. (2002). TRIZ, the altshullerian approach to solving innovation problems. In Chakrabarty, A. (Ed.), Engineering design synthesis: understanding, approaches, and tools (pp.131-148), London: Springer.

Cavallucci, D. \& Weill, R. D. (2001). Integrating Altshuller's development laws for technical systems into the design process. CIRP Annals Manufacturing Technology50(1), 115-120.

Ciruello, E., Sánchez F. \& Etxebarria, B. (2008). Compendio de definiciones del concepto innovación realizadas por autores relevantes: diseño hibrido actualizado del concepto. Dirección y Organización, 36, 61-68.

Engel, P. \& Seeijger, K. (1986). Technological and physiological characteristics of a newly developed hand-lever drive system for wheelchairs. JRRD 23(4), 37-40.

Gonzalez-Cruz, M., Aguilar-Zambrano, J., Aguilar-Zambrano, J. J. \& Gardoni, M. (2008). La estrategia de creatividad sistemática TRIZ con equipos multidisciplinares de diseño de producto. DYNA 83(6), 337-350.

Gonzalez-Cruz, M., Aguilar-Zambrano, J., Cordoba Leonor, Chamorro Cristian, Hurtado Nora, Valencia Andres, Valencia Manuel. (2009). Equipos multidisciplinares en el diseño de productos de apoyo para personas con discapacidad. Revista Ingeniería e investigación 29(3), 142-147. 
Kang, Y. J. (2004). The method for uncoupling design by contradiction matrix of TRIZ, and case study. Paper presented at the Third International Conference on Axiomatic Design, Seoul, Korea.

Lynch, N. A., Metter, E. J., Lindle, R. S., Fozard, J. L., Tobin, J. D., Roy, T. A., Fleg, J. L. \& Hurley, B. F. (1999). Muscle quality I: Age-associated differences between arm and leg muscle groups. $J$ Appl Physiol 86(1), 188-194.

Mann, D. (2002). Manufacturing technology evolution trends. Integrated Manufacturing Systems, 13(2), 86-90.

Mann, D. (2004). Fan technology: evolutionary potential and evolutionary limits. In Proceedings of the International Conference on fans (IMechE), London, UK, p. 225-234.

Moehrle, M. G. (2005) What is TRIZ? From Conceptual Basics to a Framework for Research. Creativity and Innovation Management 14(1), 313.

Pugh, S. (1991). Total design: integrated methods for successful product engineering. Wokingham, UK: Addison-Wesley Pub. Co.

Salamatov, Y. (1999). TRIZ: The right solution at the right time. The Netherlands: Insytec $\mathrm{B} . \mathrm{V}$.

Terninko, J. (1997). Step-by-step QFD customer driven product design. Boca Raton, FL: CRC Press.

Van der Woude, L., Botden, E., Vriend, I. y Veeger, D. (1997). Mechanical advantage in wheelchair lever propulsion: Effect on physical strain and efficiency.JRRD 34(3), 286-294.

Vincent, J., Bogatyreva, O., Pahl, A., Bogatyrev, N. \& Bowyer, A. (2005) Putting Biology into TRIZ: A Database of Biological Effects. Creativity and Innovation Management 14(1), 6672.

Dutta, S. (2011). The global innovation index 2011. INSEAD. Fontainebleau France 2011 